\title{
Antimicrobial stewardship and economic evaluation of urinary tract infection management in primary health care in Tunisia
}

\author{
SKANDER ESSAFI'A-G, AMEL OMEZZINE LETAIEF ${ }^{1, A, c-F}$, \\ ORCID ID: 0000-0003-0723-5884 \\ EMMA PHILLIPS ${ }^{2, \text { D-F }}$, VITTORIA VARDANEGA ${ }^{2, \text { A, c-F }}$ \\ ${ }^{1}$ Infectious Diseases Department, University Hospital Farhat Hached Sousse, Tunisia \\ ${ }^{2}$ Costello Medical, Cambridge, United Kingdom
}

A - Study Design, B - Data Collection, C - Statistical Analysis, D - Data Interpretation, E - Manuscript Preparation, F - Literature Search, G - Funds Collection

Summary Background. Inappropriate antibiotic prescriptions significantly contribute to antimicrobial resistance and are common in urinary tract infections (UTI) in primary health care. Tunisian guidelines were developed to improve UTI management for General Practitioners (GPs).

Objectives. This study aimed to evaluate GP adherence to Tunisian guidelines when prescribing antibiotics and the use of diagnostic tools for UTIs, as well as to compare current costs to those expected from guideline adherence.

Material and methods. This descriptive, cross-sectional study enrolled GPs managing patients with UTI in Sousse, Tunisia. Appropriate use of diagnostic tools and antibiotic prescriptions was evaluated. A costing model was built to compare costs between current practice and guideline adherence from a patient and country perspective for cystitis.

Results. 330 prescriptions were collected from 76 GPs. Simple cystitis was most commonly diagnosed (69.4\%). Urine dipsticks were appropriately used to diagnose $20 \%$ of cystitis cases. Urine cultures were used despite not being recommended in $45 \%$ of cystitis cases. The antibiotic prescription rate for UTI was high (92\%). Overall, $20.7 \%$ of cases adhered to the guidelines. Adherence was correlated to work in the public sector and reading the guidelines. The costing model predicted that the full adherence of antibiotic prescriptions and diagnostic tool use to cystitis guidelines could save EUR 1,698,403 annually.

Conclusions. In Tunisia, GP adherence to UTI treatment guidelines is low. Our costing model indicates guideline adherence could result in substantial savings.

Key words: anti-bacterial agents, antimicrobial stewardship, primary health care, cost-benefit analysis, urinary tract, infections.

Essafi S, Omezzine Letaief A, Phillips E, Vardanega V. Antimicrobial stewardship and economic evaluation of urinary tract infection management in primary health care in Tunisia. Fam Med Prim Care Rev 2021; 23(3): 295-300, doi: https://doi.org/10.5114/ fmpcr.2021.108193.

\section{Background}

Over the past few decades, it has been reported that antimicrobial resistance (AMR) and antimicrobial misuse are resulting in higher morbidity and mortality worldwide [1, 2]. This global public health issue is also associated with an economic burden, due to increased healthcare costs and loss of productivity [3].

In 2015, Tunisia was ranked second worldwide for consumption rate of antibiotics, with an increase in 25 daily defined doses per 1,000 inhabitants since 2000 [4]. Urinary tract infections (UTI) are some of the most common diagnoses requiring antibiotic prescriptions, representing $1.6 \%$ of consultation complaints in primary care, second only to respiratory tract infections [5]. Several strategies, known as antimicrobial stewardship (AMS), have been used to address the appropriate use of antibiotics. These include adopting a national action plan against AMR and developing national evidence-based guidelines for the management of community-acquired UTI [6, 7]. These strategies, adapted to the context of each country and their healthcare setting, are supported by interventions aiming to either enable responsible antimicrobial use (e.g. decision support tools, audit and feedback) or to restrict inappropriate or unnecessary antimicrobial use (e.g. selective reporting of susceptibility testing, formulary restrictions) [8]. While investing in AMS interventions could be costly for a healthcare system in the short term, there are clinical benefits such as reduced morbidity and cost savings in the long term [9].

\section{Objectives}

The aim of this study was to evaluate the adherence of primary care general practitioners (GPs) in Sousse, Tunisia, to the 2018 Tunisian guidelines for community-acquired UTI when prescribing antibiotics and ordering diagnostic tools (urine dipstick and cyto-bacteriological analysis). Additionally, the study aimed to compare the annual cost for patients with cystitis in Tunisia in two alternative scenarios: 1) the current practice scenario (or current scenario) within the study population, and 2) a scenario in which there is full adherence $(100 \%)$ to guidelines for both antibiotics prescriptions and diagnostic tool use, called the stewardship scenario.

\section{Material and methods}

\section{Study design and participants}

This was a descriptive, cross-sectional study that included all consenting GPs in Sousse, Tunisia, managing patients with UTI between 15 June and 15 September 2019.

Eligible patients were adults ( $\geq 18$ years of age) with a UTI diagnosis being treated by a consenting and available GP.

\section{Variables and Gyssens algorithm}

Two diagnostic tools were available to order for each UTI case: a urine dipstick, an instant tool to test for infection, indi- 
cated for cystitis, and/or a urine culture, a cytobacteriological analytic tool that tests for bacteria culture and susceptibility to antibiotics, indicated for UTIs with risk of complication.

A questionnaire was developed and administered to GPs, to be completed anonymously for every included patient. The questionnaire was composed of five sections:

- Doctor's characteristics (sector of work, years of experience, current students in training),

- Patient's characteristics (gender, age, medical history),

- Diagnosis (simple cystitis, recurrent cystitis, cystitis with risk of complication, pyelonephritis, gestational UTI, male UTI),

- Ordered tools (urine dipstick and/or urine culture)

- Initial antibiotic prescription (molecule, dose and duration),

- Issues encountered when prescribing antibiotics and/or ordering diagnostic tools.

Each antibiotic prescription was evaluated using a modified Gyssens algorithm using the following 1-5 grading system [10]:

- 5: If the molecule is not indicated for the given diagnosis,

- 4: If the right molecule is indicated, but there is a better alternative according to the guidelines,

- 3 : If there are no other alternatives, but the dose is incorrect,

- 2: If the dose is correct, but the duration is incorrect,

- 1 : If all criteria above are correct, the prescription adheres to guidelines.

The modified Gyssens algorithm was applied to every prescription manually. The resulting data was analysed using SPSS 22.0 utilising descriptive and analytical tests, including mean, univariate and multivariate analysis. Differences were statistically significant when $p \leq 0.05$.

\section{Cost scenarios development}

The relevant costs for cystitis were collected from a patient perspective, including those who were covered by health insurance, from official healthcare institution sources (hospital pharmacy, private pharmacies). These costs included antibiotics, urine culture and urine dipstick from both the public and private sector. Healthcare resource use (e.g. healthcare staff employed) was not included in the costs.

For simple and recurrent cystitis diagnoses, three antibiotics are recommended (fosfomycin, pivmecillinam, nitrofurantoin), with a similar dose and duration, allowing a single average antibiotic cost to be calculated for both diagnoses. For cystitis with risk of complication, a list of antibiotics is recommended depending on the timing of the prescription (before and after the antimicrobial susceptibility results). Their respective antibiotic costs were calculated as average between the empiric and targeted treatment cost. For all cystitis cases, the guidelines recommend urine dipsticks, which are free for the patient (the cost is included in the consultation fees), while urine culture tests are not recommended for simple cystitis and are costly for the patient.

Cystitis incidence rate and population data $[11,12]$ were used to calculate the total annual costs for all simple, recurrent and with risk of complication cystitis patients under each scenario, with the assumption that the study population is representative of the country.

The cost savings associated with adherence to the national guidelines was then calculated as the difference (both at the patient and country level) between the current practice and the stewardship scenarios. These scenarios and differences were developed within Microsoft Excel.

\section{Ethical considerations}

This study was submitted and accepted for ethical approval by the ethics committee of the University Hospital Farhat Hached Sousse, Tunisia (number 00008931).

\section{Results}

\section{Baseline patient and disease characteristics}

In total, 330 prescriptions were collected from 76 GPs. $75 \%$ $(n=57)$ of GPs were from the private sector; $63 \%(n=48)$ had over 15 years of experience. $58 \%(n=44)$ of GPs reported having read the national guidelines. The mean number of prescriptions per GP was 4.0 [2.0].

Most patients were female $(77 \%, n=256)$. The mean age was 47 years (standard deviation: 17 years). The most common comorbidities were diabetes $(20.6 \% ; n=68)$ and hypertension $(18.2 \% ; n=60) .9 .3 \%(n=31)$ of patients had an underlying urinary pathology.

Simple cystitis was the most predominant diagnosis (51.2\%; $n=169)$, followed by male UTI $(21.8 \% ; n=72)$, recurrent cystitis (12.7\%; $n=42)$, pyelonephritis $(6.1 \% ; n=20)$, cystitis with risk of complication (5.4\%; $n=18)$, gestational cystitis $(2.4 \% ; n=8)$ and asymptomatic bacteriuria $(0.3 \% ; n=1)$.

\section{Appropriate use of diagnostic tools and antibiotics}

Urine dipstick tests were appropriately ordered for $20 \%$ ( $n=46$ ) of cystitis cases. Urine cultures were ordered for $60.3 \%$ $(n=199)$ of patients. Urine cultures were appropriately ordered for $73.7 \%(n=88)$ of UTI cases with risk of complication and were inappropriately ordered for $45 \%(n=76)$ of simple cystitis cases (Table 1).

Antibiotics were prescribed in 304 cases (92\%), represented mainly by fluoroquinolones, fosfomycin, nitrofurantoin, pivmecillinam and cefixime (Figure 1). Fluoroquinolones were overall the most commonly prescribed antibiotics (42.8\%), particularly for male UTI and pyelonephritis, in $67 \%$ and $66 \%$ of cases, respectively. The prescription of fluoroquinolones in cystitis, diagnoses for which they are not indicated, varied between 33\%

\begin{tabular}{|c|c|c|c|c|c|}
\hline Diagnosis & $\begin{array}{l}\text { Dipstick only } \\
n(\%)\end{array}$ & $\begin{array}{l}\text { Urine culture only } \\
n(\%)\end{array}$ & $\begin{array}{l}\text { Dipstick and culture } \\
n(\%)\end{array}$ & $\begin{array}{l}\text { No ordered tools } \\
n(\%)\end{array}$ & \begin{tabular}{|l} 
Total \\
$n$
\end{tabular} \\
\hline Simple cystitis & $42(24.9)$ & $56(33.2)$ & $20(11.8)$ & $51(30.2)$ & 169 \\
\hline Recurrent cystitis & $4(9.5)$ & $29(69)$ & $6(14.3)$ & $3(7.2)$ & 42 \\
\hline Cystitis with risk of complication & $0(0)$ & $8(44,5)$ & $7(38,9)$ & $3(16,6)$ & 18 \\
\hline Pyelonephritis & $3(15)$ & $13(65)$ & $3(15)$ & $1(5)$ & 20 \\
\hline Gestational cystitis & $4(50)$ & $1(12,5)$ & $2(25)$ & $1(12,5)$ & 8 \\
\hline Asymptomatic bacteriuria & 0 & $1(100)$ & 0 & 0 & 1 \\
\hline Male UTI & $6(8,4)$ & $39(54,2)$ & $14(19,4)$ & $13(18)$ & 72 \\
\hline Total & 59 & 147 & 52 & 72 & 330 \\
\hline
\end{tabular}

UTI - Urinary Tract Infection 
$\square$ co-amoxiclav $\square$ cefixim $\square$ fluoroquinolones $\boxminus$ fosfomycin $\square$ nitrofurantoin $\square$ Other

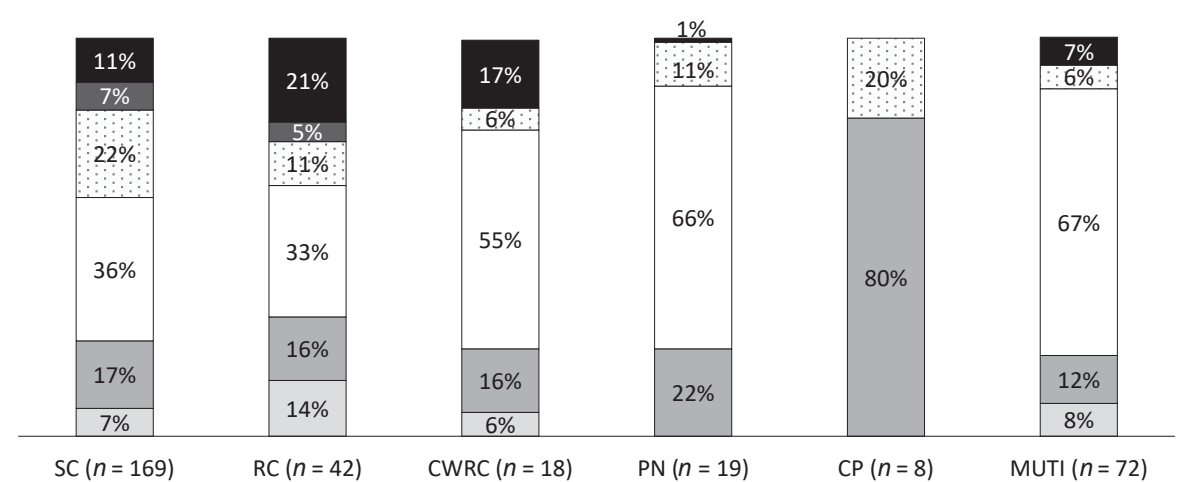

Figure 1. Antibiotic prescription trends for different UTI diagnoses

CP - Cystitis in Pregnancy, CWRC - Cystitis with Risk of Complication, MUTI - Male Urinary Tract Infection, PN - Pyelonephritis, SC - Simple Cystitis, UTI - Urinary Tract Infection.

\begin{tabular}{|c|c|c|c|c|c|c|}
\hline Score, $n(\%)$ & 1 & 2 & 3 & 4 & 5 & Total \\
\hline $\begin{array}{l}\text { Cystitis } \\
\text { simple cystitis } \\
\text { recurrent cystitis } \\
\text { cystitis with risk of complication }\end{array}$ & $\begin{array}{l}50(24.0) \\
45(28.6) \\
5(14.3) \\
0(0)\end{array}$ & $\begin{array}{l}3(1.4) \\
2(1.3) \\
0(0) \\
1(5.8)\end{array}$ & $\begin{array}{l}6(2.9) \\
3(1.9) \\
2(5.7) \\
1(5.8)\end{array}$ & $\begin{array}{l}\text { NA } \\
\text { NA } \\
\text { NA } \\
\text { NA }\end{array}$ & $\begin{array}{l}150(71.8) \\
107(68.2) \\
28(80) \\
15(88.2)\end{array}$ & $\begin{array}{l}209 \\
157 \\
35 \\
17\end{array}$ \\
\hline Pyelonephritis & $3(17.6)$ & $9(52.9)$ & $2(11.7)$ & NA & $3(17.6)$ & 17 \\
\hline Gestational cystitis & $2(25)$ & NA & NA & $5(62.5)$ & $1(12.5)$ & 8 \\
\hline Male UTI & $8(11.4)$ & $27(38.6)$ & $9(12.8)$ & $1(1.4)$ & $25(35.7)$ & 70 \\
\hline Total & $63(20.7)$ & $39(12.8)$ & $17(5.6)$ & $6(2)$ & $179(58.9)$ & 304 \\
\hline
\end{tabular}

NA - Not available, UTI - Urinary Tract Infection.

\begin{tabular}{|l|l|l|l|}
\hline Table 3. Cost per research population & Current scenario cost (TND) & Stewardship scenario (TND) & Stewardship cost savings (TND) \\
\hline Simple cystitis & 2,417 & 2,171 & 246 \\
\hline Antibiotic & 0 & 0 & 0 \\
\hline Dipstick* & 1,613 & 0 & 1,613 \\
\hline Urine culture & 4,030 & 2,171 & 1,859 \\
\hline Total cost & Current scenario cost (TND) & Stewardship scenario (TND) & Stewardship cost savings (TND) \\
\hline Recurrent cystitis & 783 & 540 & 243 \\
\hline Antibiotic & 0 & 0 & 0 \\
\hline Dipstick* & 650 & 804 & -155 \\
\hline Urine culture & 1,433 & 1,344 & 89 \\
\hline Total cost & Current scenario cost (TND) & Stewardship scenario (TND) & Stewardship cost savings (TND) \\
\hline Cystitis with risk of complication & 443 & -90 \\
\hline Antibiotic & 353 & 0 & 0 \\
\hline Dipstick* & 0 & 409 & -55 \\
\hline Urine culture & 355 & 852 & -144 \\
\hline Total cost & 708 & & \\
\hline
\end{tabular}

* Dipstick cost is free for patients, TND - Tunisian Dinar.

and $55 \%$. Fosfomycin, which is prescribed for cystitis, was prescribed for between $6 \%$ and $22 \%$ of all types of cystitis cases. Nitrofurantoin was prescribed in $7 \%$ and $5 \%$ of simple cystitis and recurrent cystitis cases, respectively, and is indicated for both diagnoses. Pivmecillinam was not prescribed for any cystitis case, despite being indicated for these diagnoses. Cefixime was prescribed mainly for cystitis in pregnancy (80\%), as recommended. However, in $16-17 \%$ of cases, it was inappropriately prescribed for other types of cystitis.

Overall, $20.7 \%$ of antibiotic prescriptions adhered to the national guidelines; adherence was $24 \%$ for all cystitis cases (Table 2). There was an association between adherence to guidelines and the public sector, reading the guidelines, current students in training, more than 15 years of practice experience and the female gender of patients. Multivariate analysis demonstrated statistically significant correlations between guideline adherence and working in the public sector $(p=0.008)$ or reading the guidelines $(p=0.001)$.

\section{Costing model}

In the costing model, a saving of Tunisian Dinar (TND) 1,859 was estimated for simple cystitis in the stewardship scenario (Table 3 ). This mainly represented a saving in urine culture costs. The results showed a smaller cost savings with the stewardship 


\begin{tabular}{|l|l|l|l|}
\hline \multicolumn{4}{|l}{ Table 4. Costing model results per patient and annual cost for Tunisia for cystitis management } \\
\hline Simple cystitis & Current scenario cost (TND) & Stewardship scenario (TND) & Stewardship cost savings (TND) \\
\hline Antibiotic & 14 & 13 & 1 \\
\hline Dipstick & 0 & 0 & 0 \\
\hline Urine culture & 10 & 0 & 10 \\
\hline Total cost & 24 & 13 & 11 \\
\hline Recurrent cystitis & Current scenario cost (TND) & Stewardship scenario (TND) & Stewardship cost savings (TND) \\
\hline Antibiotic & 19 & 13 & 6 \\
\hline Dipstick & 0 & 0 & 0 \\
\hline Urine culture & 15 & 19 & -4 \\
\hline Total cost & 34 & 32 & 2 \\
\hline Cystitis with risk of complication & Current scenario cost (TND) & Stewardship scenario (TND) & Stewardship cost savings (TND) \\
\hline Antibiotic & 20 & 25 & -5 \\
\hline Dipstick & 0 & 0 & 0 \\
\hline Urine culture & 20 & 23 & -3 \\
\hline Total cost & 39 & 47 & -8 \\
\hline Annual cost per country (cystitis) & Current scenario cost (TND) & Stewardship scenario (TND) & Stewardship cost savings (TND) \\
\hline Antibiotic & $22,649,113$ & $20,264,084$ & $2,385,029$ \\
\hline Dipstick & 0 & 0 & 0 \\
\hline Urine culture & $5,486,184$ & $2,542,889$ & $2,943,295$ \\
\hline Total cost & $28,135,296$ & $22,806,973$ & $5,328,323$ \\
\hline & & & \\
\hline
\end{tabular}

TND - Tunisian Dinar.

scenario for recurrent cystitis (TND 89) and no cost-saving for cystitis with risk of complication (TND144).

For a simple cystitis episode, the model estimated an average saving of TND 11 (TND 1 saved for antibiotics and TND 10 for urine culture) for a patient in the stewardship scenario (Table 4). When calculated for the whole population of Tunisia, the model estimated that the patient cost per year for the total population under the current scenario is TND 28,135,296, compared with a cost of TND 22,806,973 under the stewardship scenario with full adherence to national guidelines. Overall, the model estimated that full adherence leads to a cost savings of TND 5,328,323 (approximately EUR 1,698,403 using current exchange rates; Table 4).

\section{Discussion}

\section{Main findings}

This study aimed to evaluate real-life UTI management in primary health care compared to national guidelines, as well as the direct economic implications from a patient and country perspective for cystitis.

A low rate of adherence to guidelines was found (20.7\%). The economic model demonstrated that it was cost saving to adopt a stewardship scenario for cystitis management (TND 5,328,323).

\section{Antibiotic prescriptions and conformity to recom- mendations}

Despite the high rate of cystitis in our findings (69\%), we noted low prescriptions of fosfomycin (16.4\%) and nitrofurantoin (5.2\%) and no prescription of pivmecillinam; these antibiotics representing the first line of treatment for cystitis, compared to quinolones, which are not recommended and were prescribed in $44 \%$ of cases.

A Danish study in 2019 (Holm et al.) in primary health care showed that pivmecillinam was the most prescribed antibiotic for lower acute UTI (45.8\%), followed by sulfonamides $(27 \%)$; both antibiotics being the first-line recommended treatment, as well as quinolones (19.7\%) [13].
The prescription of fluoroquinolones is not indicated in cystitis, despite its large antimicrobial action, due to the high risk of resistance development [14]. Nonetheless, we noted a high rate of fluoroquinolones prescriptions (46.5\%), particularly for cystitis.

Kobayashi et al. conducted a retrospective study on outpatient visits in the USA regarding UTI from 2002 to 2011 and found that out of 7111 visits, about $80 \%$ were treated with antibiotics, and almost half was fluoroquinolones [15]. This concern regarding the high use of fluoroquinolones does not only result from an ecological standpoint. Noting its ease of dosing, expanded spectrum of activity and favourable pharmacokinetics, quinolones represent the most prescribed antibiotics in ambulatory care [16].

The Gyssens algorithm was selected to evaluate antibiotic prescriptions, as it is simple to use. In a French study conducted in 2011, using a similar algorithm, results showed $20 \%$ adherence to guidelines for UTI management. A Spanish study conducted in 2011 found that only $17.7 \%$ of antibiotic prescriptions for cystitis and $17.5 \%$ of antibiotic prescriptions for complicated cystitis conformed to guidelines [18].

\section{Antibiotic prescription drivers}

Poor adherence to guidelines has been linked to the habits of the GPs, their training and post-graduate education [19]. However, other factors can explain this low rate, including healthcare system management, available resources (antibiotics and diagnostic tools), the culture of antibiotic consumption, as well as patients' opinions and healthcare coverage having an impact on shared medical decisions between the patient and the GP [19]. A systematic review concluded that prescription behaviour is a complex process based on a range of internal and external factors [20]. Dominant among these is the prescriber's perception that patients want antibiotics and the fear of what might happen if antibiotics are not forthcoming [20].

The use of fluoroquinolones is an example of the high rates of inappropriate prescriptions associated with treatment availability, efficiency and a wide spectrum of activity [14]. 
In our study, the association between adhering to guidelines and reading the guidelines or training students was statistically significant $(p \leq 0.0001)$. This suggests it is important to endorse AMS interventions and monitor their impact on GP practices. This could begin with implementation of training during the first years of medical schools through to post-graduate training courses. Once GPs are qualified and practicing, continued AMS education could be implemented though continuous professional development with certified training, online courses and access to decision-making tools [21]. In the UK, the 'Stemming the Tide of Antibiotic Resistance' (STAR) programme has been developed to build on behaviour change theories, integrating a blend of learning techniques online and within the general practice setting, including promotion of the evidence base and reflection on practice [22]. The impact was demonstrated by the GPs in their feedback on self-reported improvement in knowledge [23]. A randomised controlled trial showed a reduction of $4 \%$ in overall prescription of antibiotics in practices receiving the STAR training package [24].

\section{Costing model implications}

The costing model developed compares the costs for cystitis management from a patient perspective, including diagnostic tests and antibiotics, under a current practice scenario and a stewardship scenario. The stewardship scenario aligned with the Tunisian guidelines for UTI management and allowed for an estimation of the cost for the patients if there was $100 \%$ adherence to the guidelines when prescribing antibiotics for female patients with cystitis.

This model only considered cystitis management, as cystitis diagnoses were found to be the most common diagnoses and had clear AMS guidelines. The results demonstrated a clear cost savings with AMS, particularly for simple cystitis (the most common diagnosis, with $69 \%$ out of all diagnoses). These savings were largely found in diagnostic tools (saving TND 1,613 in the research population) but also in antibiotics (saving TND 246 in the research population).

This model can be duplicated for any primary care setting, for UTI or any other common infection (e.g. skin or respiratory infection) considering the diagnosis tools, recommended antibiotics and their relevant costs, as well as if there are official guidelines that can be used as reference to build a stewardship scenario.

Other AMS programmes have shown long-term economic benefits for patients, with a $33.9 \%$ reduction in antibiotic costs for all types of infections and a $4.5 \%$ reduction in the cost of bacterial infections after the implementation of the AMS programme $[25,26]$. However, these studies were completed in hospital settings and took into consideration duration of hospital stay, drug resistance and antibiotic costs over a longer time period than the current study. There is very little evidence concerning the economic evaluation of AMS in a primary care setting, particularly for UTIs. Gillespie et al. published a casecontrol study in 2016 between two intervention arms of AMS programmes for UTI management and one control arm. The study compared several types of costs for patients (intervention set-up, audit and feedback, GP visits, laboratory tests, antimicrobial prescriptions), finding a cost per patient of EUR 84.20, 88.72 and 67.00 for the two intervention arms and control arm, respectively [27]. This difference in cost may not be comparable, but the context is similar to this study. Further longitudinal primary healthcare studies are needed to measure the impact of AMS programmes, such as the number of GP revisits, drugresistant infections, need for hospitalisation, morbidity and mortality.

\section{Conclusions}

In the fight against antimicrobial resistance, AMS represents a series of public health interventions. In Tunisia, guidelines have been developed to address the management of UTIs in primary health care, but few initiatives are in place to monitor the impact of their implementation. This study found that adherence to guidelines for UTI in primary care is low and may be improved by increasing recommended antibiotic availability and providing training for GPs. Furthermore, a costing model estimated that substantial savings could be made through guideline adherence, including using the recommended antibiotics (molecule, dose and duration) and diagnostic tool (dipstick and/or urine culture). This costing model can be used freely and duplicated for any primary care setting, for UTI or any other common infection where guidelines are available. In this way, it could serve as an advocacy tool to encourage healthcare authorities to invest and implement effective AMS interventions through demonstrating the possible economic benefits.

Acknowledgments. The authors thank the patients and healthcare professionals who participated in this study. The authors also acknowledge Declan Summers, Elizabeth Evans and Shahad Atrah, Costello Medical, UK, for their input in the development of the economic model.

Source of funding: Data collection was done using the personal financial means of the corresponding authors. The economic model was developed during a Global Health Internship opportunity offered by Costello Medical, UK, for the period January-March 2020.

Conflicts of interest: Emma Phillips and Vittoria Vardanega are employees of Costello Medical, UK. Skander Essafi and Amel OmezzineLetaief have no conflicts of interest to declare.

\section{References}

1. Goossens H, Ferech M, Stichele RV, et al. Outpatient antibiotic use in Europe and association with resistance: a cross-national database study. Lancet 2005; 365: 579-587.

2. Bell BG, Schellevis F, Stobberingh E, et al. A systematic review and meta-analysis of the effects of antibiotic consumption on antibiotic resistance. BMC Infect Dis 2014; 14: 13, doi: 10.1186/1471-2334-14-13.

3. ECDC/EMEA Joint Working Group. Technical Report. The bacterial challenge, time to react: a call to narrow the gap between multidrugresistant bacteria in the EU and the development of new antibacterial agents. Stockholm: ECDC; 2009.

4. Klein EY, Van Boeckel TP, Martinez EM, et al. Global increase and geographic convergence in antibiotic consumption between 2000 and 2015. Proc Natl Acad Sci 2018; 115: E3463-E3470.

5. Gataa R, Nabli Ajmi T, Bougmiza I, et al. La morbidité diagnostiquée en première ligne dans la région sanitaire de Sousse (Tunisie). Rev Med Brux 2008; 29: 471-480.

6. Ministry of Health Tunisia. Plan d'Action Nationale conte la résistance aux antibactériens 2019. Available from URL: http://www.santetunisie.rns.tn/images/docs/anis/actualite/2019/dec/PAN-TUNISIE-2019-2023.pdf.

7. Société Tunisienne de Pathologies Infectieuses. Recommandations de prise en charge des infections urinaires communautaires 2018. Available from URL: https://www.infectiologie.org.tn/pdf_ppt_docs/recommandations/1527103156.pdf.

8. Davey P, Marwick CA, Scott CL, et al. Interventions to improve antibiotic prescribing practices for hospital inpatients. Cochrane Database Syst Rev 2017; 2, doi: 10.1002/14651858.CD003543. 
9. Huebner C, Flessa S, Huebner N-O. The economic impact of antimicrobial stewardship programmes in hospitals: a systematic literature review. J Hosp Infect 2019; 102: 369-376.

10. Gyssens IC. Quality measures of antimicrobial drug use. Int J Antimicrob Agents 2001; 17: 9-19.

11. World Bank. Total Population of Tunisia 2019 [cited 08.02.2020]. Available from URL: https://data.worldbank.org/indicator/SP.POP. TOTL?locations=TN.

12. Lobel B, Soussy C. Les infections urinaires [cited 03.12.2019]. Available from URL: https://www.eyrolles.com/Sciences/Livre/les-infections-urinaires-9782287251726/.

13. Holm A, Cordoba G, Aabenhus R. Prescription of antibiotics for urinary tract infection in general practice in Denmark. Scand J Prim Health Care 2019; 37: 83-89.

14. Aldred KJ, Kerns RJ, Osheroff N. Mechanism of quinolone action and resistance. Biochemistry 2014; 53: 1565-1574.

15. Kobayashi M, Shapiro DJ, Hersh AL, et al. Outpatient antibiotic prescribing practices for uncomplicated urinary tract infection in women in the United States, 2002-2011. Open Forum Infect Dis 2016; 3(3): ofw159, doi: 10.1093/ofid/ofw159.

16. Kabbani S, Hersh AL, Shapiro DJ, et al. Opportunities to improve Fluoroquinolone prescribing in the United States for adult ambulatory care visits. Clin Infect Dis 2018; 67(1): 134-136, doi: 10.1093/cid/ciy035.

17. Denes E, Prouzergue J, Ducroix-Roubertou S, et al. Antibiotic prescription by general practitioners for urinary tract infections in outpatients. Eur J Clin Microbiol Infect Dis 2012; 31: 3079-3083.

18. Llor C, Rabanaque G, López A, et al. The adherence of GPs to guidelines for the diagnosis and treatment of lower urinary tract infections in women is poor. Fam Pract 2011; 28: 294-299.

19. Haute Autorité de santé. Guide méthodologique. Méthodes quantitatives pour évaluer les interventions visant à améliorer les pratiques. 2007. Available from URL: https://www.has-sante.fr/upload/docs/application/pdf/eval_interventions_ameliorer_pratiques_guide.pdf.

20. Teixeira Rodrigues A, Roque F, Falcão A, et al. Understanding physician antibiotic prescribing behaviour: a systematic review of qualitative studies. Int J Antimicrob Agents 2013; 41: 203-212.

21. Pulcini C, Gyssens IC. How to educate prescribers in antimicrobial stewardship practices. Virulence 2013; 4: $192-202$.

22. Simpson SA, Butler CC, Hood K, et al. Stemming the Tide of Antibiotic Resistance (STAR): a protocol for a trial of a complex intervention addressing the 'why' and 'how' of appropriate antibiotic prescribing in general practice. BMC Fam Pract 2009; 10: 20, doi: 10.1186/1471-2296-10-20.

23. Bekkers MJ, Simpson SA, Dunstan F, et al. Enhancing the quality of antibiotic prescribing in Primary Care: qualitative evaluation of a blended learning intervention. BMC Fam Pract 2010; 11: 34, doi: 10.1186/1471-2296-11-34.

24. Butler CC, Simpson SA, Dunstan F, et al. Effectiveness of multifaceted educational programme to reduce antibiotic dispensing in primary care: practice based randomised controlled trial. BMJ 2012; 344: d8173-d8173.

25. OECD. Stemming the superbug tide: just a few dollars more. OECD Health Policy Studies. Paris: OECD Publishing; 2018, doi: 10.1787/9789264307599-en.

26. Cost-effectiveness of policies to limit antimicrobial resistance in Dutch Healthcare Organisations - Governmental Report. Available from URL: https://www.government.nl/documents/reports/2016/01/27/cost-effectiveness-of-policies-to-limit-antimicrobial-resistance-in-dutch-healthcare-organisations.

27. Gillespie $P$, Callan $A, O$ 'Shea $E$, et al. The cost effectiveness of the SIMPle intervention to improve antimicrobial prescribing for urinary tract infection in primary care. J Public Health (Oxf) 2017; 39(4): e282-e289, doi: 10.1093/pubmed/fdw102.

Tables: 4

Figures: 1

References: 27

Received: 19.01.2021

Reviewed: 10.02 .2021

Accepted: 08.03.2021

Address for correspondence:

Skander Essafi, MD

Infectious Diseases Department

University Hospital Farhat Hached Sousse

Sousse 4000 Ibn Jazzar Street

Tunisia

Tel.: 0021625346515

E-mail: skander.es@gmail.com 